\title{
Numerical Integration Constants
}

Tables of the $b_{j}$ and $x_{j}$ in the approximate quadrature formula

$$
\int_{0}^{1} x^{n} g(x) d x \doteq \sum_{j=1}^{m} b_{j} g\left(x_{j}\right)
$$

are given for $n=0(1) 5, m=1(1) 8$ to $12 \mathrm{D}$. The $x_{j}$ are the zeros of certain orthonormal polynomials $q_{m}(x)$; the $q_{m}(x)$ are given explicitly for the same range of $n, m$. It is well known that there is equality in (1) for polynomials not exceeding degree $2 m-1$. These tables extend those given by Hammer, Marlowe, and Stroud [2].

P. C. Hammer points out that due to the relation,

$$
\int_{c}^{d}\left\{\left(\int_{c}^{t}\right)^{n} f(t)(d t)^{n}\right\} d t=\frac{(d-c)^{n+1}}{n !} \int_{0}^{1} x^{n} f(d-[d-c] x) d x
$$

(see [4]), the tables given below can be applied to the evaluation of repeated integrals.

$$
\boldsymbol{n}=\mathbf{0}
$$

$m$

$0 \quad q_{0}(x)=\sqrt{1}$

$1 \quad q_{1}(x)=\sqrt{3}(1-2 x)$

$2 \quad q_{2}(x)=\sqrt{5}\left(1-6 x+6 x^{2}\right)$

$3 \quad q_{3}(x)=\sqrt{7}\left(1-12 x+30 x^{2}-20 x^{3}\right)$

$4 \quad q_{4}(x)=\sqrt{9}\left(1-20 x+90 x^{2}-140 x^{3}+70 x^{4}\right)$

$5 \quad q_{5}(x)=\sqrt{11}\left(1-30 x+210 x^{2}-560 x^{3}+630 x^{4}-252 x^{5}\right)$

$6 \quad q_{0}(x)=\sqrt{13}\left(1-42 x+420 x^{2}-1680 x^{3}+3150 x^{4}-2772 x^{5}+924 x^{6}\right)$

$7 \quad q_{7}(x)=\sqrt{15}\left(1-56 x+756 x^{2}-4200 x^{3}+11550 x^{4}-16632 x^{5}+12012 x^{6}\right.$ $\left.-3432 x^{7}\right)$

8

$$
\begin{aligned}
q_{8}(x)= & \sqrt{17}\left(1-72 x+1260 x^{2}-9240 x^{8}+34650 x^{4}-72072 x^{5}+84084 x^{6}\right. \\
& \left.-51480 x^{7}+12870 x^{8}\right) .
\end{aligned}
$$

$n=1$

$0 \quad q_{0}(x)=\sqrt{2}$

$1 \quad q_{1}(x)=\sqrt{4}(2-3 x)$

$2 \quad q_{2}(x)=\sqrt{6}\left(3-12 x+10 x^{2}\right)$

$3 \quad q_{3}(x)=\sqrt{8}\left(4-30 x+60 x^{2}-35 x^{3}\right)$

$4 \quad q_{4}(x)=\sqrt{10}\left(5-60 x+210 x^{2}-280 x^{3}+126 x^{4}\right)$

$5 \quad q_{5}(x)=\sqrt{12}\left(6-105 x+560 x^{2}-1260 x^{3}+1260 x^{4}-462 x^{5}\right)$

$6 \quad q_{6}(x)=\sqrt{14}\left(7-168 x+1260 x^{2}-4200 x^{3}+6930 x^{4}-5544 x^{5}+1716 x^{6}\right)$

$7 \quad q_{7}(x)=\sqrt{16}\left(8-252 x+2520 x^{2}-11550 x^{3}+27720 x^{4}-36036 x^{5}\right.$

$\begin{aligned} & \left.+24024 x^{6}-6435 x^{7}\right) \\ q_{8}(x)= & \sqrt{18}\left(9-360 x+4620 x^{2}-27720 x^{8}+90090 x^{4}-168168 x^{5}\right. \\ & \left.+180180 x^{6}-102960 x^{7}+24310 x^{8}\right)\end{aligned}$

8 


$$
\begin{aligned}
& 0 \quad q_{0}(x)=\sqrt{3} \\
& n=2 \\
& 1 \quad q_{1}(x)=\sqrt{5}(3-4 x) \\
& 2 \quad q_{2}(x)=\sqrt{7}\left(6-20 x+15 x^{2}\right) \\
& 3 \quad q_{3}(x)=\sqrt{9}\left(10-60 x+105 x^{2}-56 x^{3}\right) \\
& 4 \quad q_{4}(x)=\sqrt{11}\left(15-140 x+420 x^{2}-504 x^{3}+210 x^{4}\right) \\
& 5 \quad q_{5}(x)=\sqrt{13}\left(21-280 x+1260 x^{2}-2520 x^{3}+2310 x^{4}-792 x^{5}\right) \\
& 6 \quad q_{6}(x)=\sqrt{15}\left(28-504 x+3150 x^{2}-9240 x^{3}+13860 x^{4}-10296 x^{5}\right. \\
& \left.+3003 x^{6}\right) \\
& 7 \quad q_{7}(x)=\sqrt{17}\left(36-840 x+6930 x^{2}-27720 x^{3}+60060 x^{4}-72072 x^{5}\right. \\
& \left.+45045 x^{6}-11440 x^{7}\right) \\
& 8 \quad q_{8}(x)=\sqrt{19}\left(45-1320 x+13860 x^{2}-72072 x^{3}+210210 x^{4}-360360 x^{5}\right. \\
& \left.+360360 x^{6}-194480 x^{7}+43758 x^{8}\right) \\
& 0 \quad q_{0}(x)=\sqrt{4} \\
& n=3 \\
& 1 \quad q_{1}(x)=\sqrt{6}(4-5 x) \\
& 2 \quad q_{2}(x)=\sqrt{8}\left(10-30 x+21 x^{2}\right) \\
& 3 \quad q_{3}(x)=\sqrt{10}\left(20-105 x+168 x^{2}-84 x^{3}\right) \\
& 4 \quad q_{4}(x)=\sqrt{12}\left(35-280 x+756 x^{2}-840 x^{3}+330 x^{4}\right) \\
& 5 \quad q_{5}(x)=\sqrt{14}\left(56-630 x+2520 x^{2}-4620 x^{3}+3960 x^{4}-1287 x^{5}\right) \\
& 6 \quad q_{6}(x)=\sqrt{16}\left(84-1260 x+6930 x^{2}-18480 x^{3}+25740 x^{4}-18018 x^{5}\right. \\
& \left.+5005 x^{6}\right) \\
& 7 \quad q_{7}(x)=\sqrt{18}\left(120-2310 x+16632 x^{2}-60060 x^{3}+120120 x^{4}-135135 x^{5}\right. \\
& \left.+80080 x^{6}-19448 x^{7}\right) \\
& 8 q_{8}(x)=\sqrt{20}\left(165-3960 x+36036 x^{2}-168168 x^{3}+450450 x^{4}-720720 x^{5}\right. \\
& \left.+680680 x^{6}-350064 x^{7}+75582 x^{8}\right) \\
& 0 \quad q_{0}(x)=\sqrt{5} \\
& n=4 \\
& 1 \quad q_{1}(x)=\sqrt{7}(5-6 x) \\
& 2 \quad q_{2}(x)=\sqrt{9}\left(15-42 x+28 x^{2}\right) \\
& 3 \quad q_{3}(x)=\sqrt{11}\left(35-168 x+252 x^{2}-120 x^{3}\right) \\
& 4 \quad q_{4}(x)=\sqrt{13}\left(70-504 x+1260 x^{2}-1320 x^{3}+495 x^{4}\right) \\
& 5 \quad q_{5}(x)=\sqrt{15}\left(126-1260 x+4620 x^{2}-7920 x^{3}+6435 x^{4}-2002 x^{5}\right) \\
& 6 \quad q_{6}(x)=\sqrt{17}\left(210-2772 x+13860 x^{2}-34320 x^{3}+45045 x^{4}-30030 x^{5}\right. \\
& \left.+8008 x^{6}\right) \\
& 7 \quad q_{7}(x)=\sqrt{19}\left(330-5544 x+36036 x^{2}-120120 x^{3}+225225 x^{4}-240240 x^{6}\right. \\
& \left.+136136 x^{6}-31824 x^{7}\right) \\
& 8 q_{8}(x)=\sqrt{21}\left(495-10296 x+84084 x^{2}-360360 x^{3}+900900 x^{4}\right. \\
& \left.-1361360 x^{5}+1225224 x^{6}-604656 x^{7}+125970 x^{8}\right) \\
& n=5 \\
& 0 \quad q_{0}(x)=\sqrt{6} \\
& 1 \quad q_{1}(x)=\sqrt{8}(6-7 x) \\
& 2 \quad q_{2}(x)=\sqrt{10}\left(21-56 x+36 x^{2}\right) \\
& 3 \quad q_{3}(x)=\sqrt{12}\left(56-252 x+360 x^{2}-165 x^{3}\right) \\
& 4 \quad q_{4}(x)=\sqrt{14}\left(126-840 x+1980 x^{2}-1980 x^{3}+715 x^{4}\right) \\
& 5 \quad q_{5}(x)=\sqrt{16}\left(252-2310 x+7920 x^{2}-12870 x^{3}+10010 x^{4}-3003 x^{5}\right)
\end{aligned}
$$




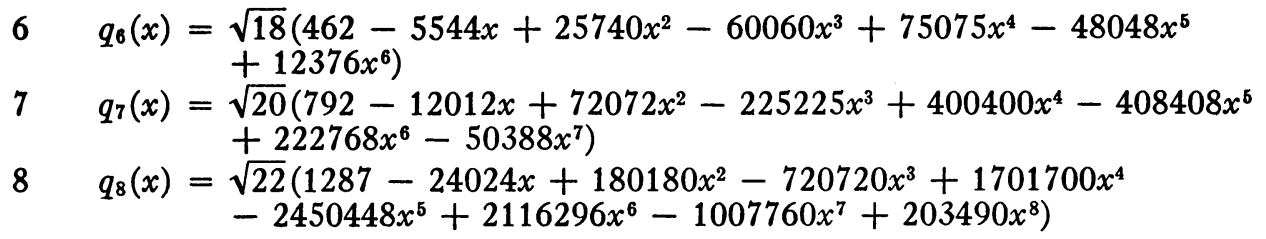

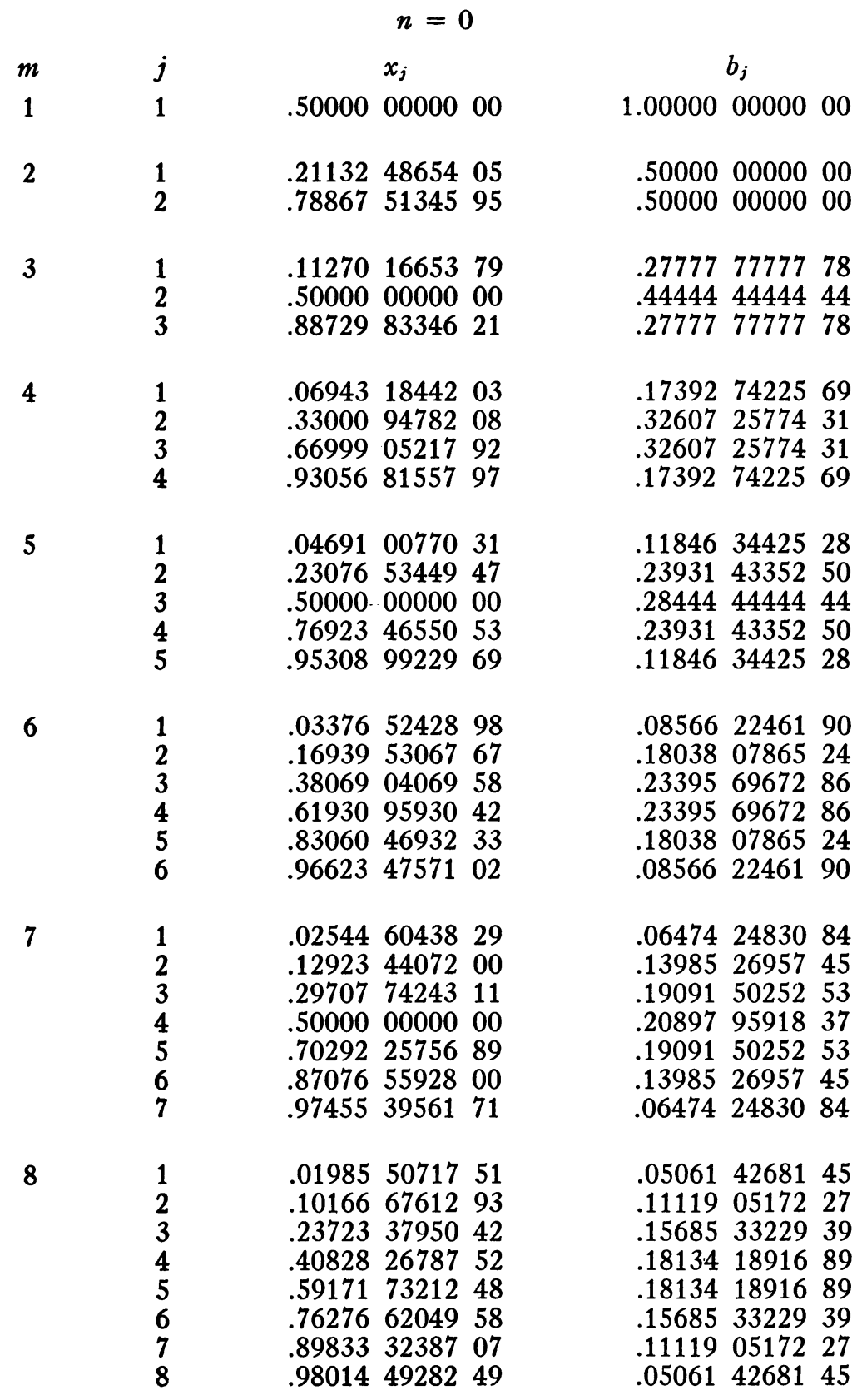


NUMERICAL INTEGRATION CONSTANTS

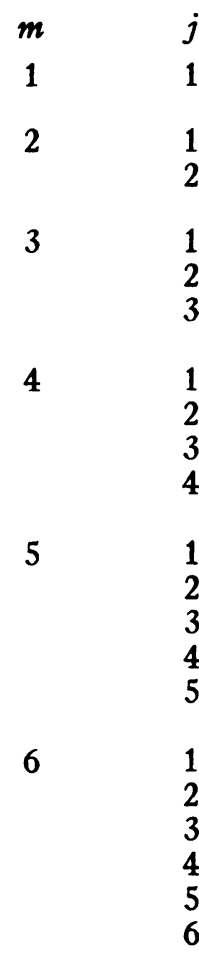

$$
n=1
$$

$x_{j}$

$b_{j}$

.500000000000

.181958618256

.318041381744

$\begin{array}{lll}.35505 & 10257 & 22 \\ .84494 & 89742 & 78\end{array}$

.069826979901

.229241106360

.200931913739

.590533135559

.911412040487

.031180970950

.129847547608

$\begin{array}{llll}20346 & 45680 & 10\end{array}$

$.1355069134 \quad 31$

.723156986362

.942895803885

.015747914522

.098535085799

.304535726646

.562025189753

.801986582126

.960190142949

.073908870073

.146386987085

.167174638094

$.0967815902 \quad 27$

.073054328680

.230766137970

.441328481228

.663015309719

.851921400332

.970683572840

7

$.05626 \quad 2560537$

.180240691737

$\begin{array}{llll}.35262 & 47171 & 13\end{array}$

$\begin{array}{llll}.54715 & 36263 & 31\end{array}$

$\begin{array}{llll}.73421 & 01772 & 15\end{array}$

.885320946839

.977520613561

.008738301814

.043955165551

.098661150891

.140792553788

.135542497232

.072310330726

.005214362203

.027408356722

.066384696465

.107125065696

.127390897300

.110509258191

.055967363423

8

.044633955290

$.14436 \quad 6257042$

.286824757144

.454813315197

$.62806 \quad 78354 \quad 17$

.785691520604

.908676392100

$.9822200848 \quad 53$

.003295191442

.017842902656

.045439319505

.079199599492

.106047359436

.112505799471

.091119023636

.044550804362

$$
n=2
$$

$\begin{array}{lll}1 & 1 & .750000000000\end{array}$

.333333333333

.455848155989

$.8774851773 \quad 45$

.100785882080

.232547451254

3

.294997790112

.652996233962

.029950703009

.146246269260 .157136361065 
$m$

4

5

6

7

8

2

3

4

5 $x_{j}$

.204148582103

.482952704896

.761399262448

.951499450553

.148945787053

.365666527369

.610113612934

.826519679228

.965421060082

.113194383822

.284318872688

.490963586835

.697563081977

.868436058342

.974095444906

$.0888168334 \quad 37$

.226482753409

.399978486721

$.5859978554 \quad 03$

.759445873952

.896910970852

.979867226227

.071491035040

.184228296417

.330447728176

.494402921816

.658348008523

.804524831511

.917099382514

.983902240448

$$
n=3
$$

.800000000000

.529857935895

.898713492677

.363264630217

.698811269164

.937924100620

.261477788831

.535846446088

.790283229969

.957847080566

.196212007397

.417100211822

$\begin{array}{llll}64857 & 00042 \quad 37\end{array}$

.845605149974

.969435703493 $b_{j}$

.010352240750

.068633887173

.143458789799

.110888415611

.004113825203

.032055600723

.089200161222

.126198961900

.081764784286

$\begin{array}{lll}.00183 & 10758 \quad 07\end{array}$

$\begin{array}{lll}.01572 & 0297185\end{array}$

.051289571130

.094577186749

.107376499737

.062538702727

.000892688034

.008162925632

.029422211290

.063146378709

.091733803280

.090698824613

.049276501776

.000468517784

$.0044745217 \quad 13$

.017246863780

.040814426389

.068447183422

.085284769172

.076818093267

.039778957807

.250000000000

$.0669052498 \quad 07$

.183094750193

.016479059283

.104599897557

.128921043161

.004658367060

.042541724143

.109004368939

.093795539859

.001520689371

.016957324863

.060444953204

.100316504465

.070760528097 


$$
x_{j}
$$

$b_{j}$

.152273161784

$\begin{array}{llll}.33130 & 04570 & 38\end{array}$

.532411566729

$.7256027783 \quad 30$

.881616684437

.976795351682

$.1214271288 \quad 32$

.268363440311

.440866460623

.618604028432

.780253551966

.906362534145

.981769914514

8

$.0990017577 \quad 10$

$.2212435073 \quad 50$

.369123900012

.528545431202

.683993248432

.820283949679

.924093712899

.985293440085

$$
n=4
$$

.833333333333

$\begin{array}{lll}.58633 & 6582323\end{array}$

.913663417677

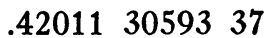

.733889355208

.945997585455

4

$\begin{array}{lll}.31213 & 54928 & 47\end{array}$

.578915659562

.812891516616

.962723997641

5

$\begin{array}{lll}23979 & 2044802\end{array}$

.460933674532

.680059232741

.860886343676

.972614418534

6

$\begin{array}{lll}1894695839 & 22\end{array}$

$\begin{array}{lll}.37275 & 11560 & 14\end{array}$

$\begin{array}{llll}.56757 & 23728 & 55\end{array}$

.748836497506

$.89238 \quad 51584 \quad 47$

.978985231256

.000561710874

$.00708 \quad 5315932$

.030526192226

.068443281768

$.08830 \quad 0991241$

.055082507960

.000229904127

.003147596407

.015312167126

.040995168604

069750098107

.076556561363

.044008504265

.000102460078

.001485684083

.007855073841

.023631580668

$.04745 \quad 43798 \quad 12$

.067361839393

.066182035322

.035926946803

.200000000000

.049082492278 .150917507722

.010469042183 .080276673456 .109254284361

.002516351647 .029169382162 .087067712064 .081246554127

.000696977078 .010210541725 .044024469505 .082712713102 .062355298589

.000219413995 .003726784439 .019956264693 .052239954293 $.0746491503 \quad 13$ .049208432267 


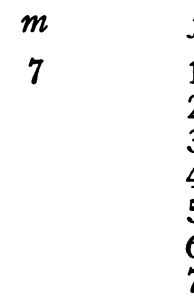

8

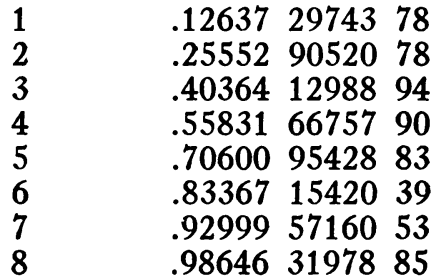

$$
n=5
$$

1

2

3

4 $\begin{array}{lll}.15324 & 14388 & 69 \\ .30632 & 65225 & 42 \\ .47654 & 00930 & 01 \\ .64638 & 93025 & 20 \\ .79771 & 66898 & 15 \\ .91421 & 99005 & 65 \\ .98334 & 38304 & 67\end{array}$

8

1

.857142857143

.630791593830

.924763961726

.467983235455 .761623969699 .952210976664

.356893729050 .614669389855 .831079003860 .966588646465

5

.279693124812 .498709827030 $\begin{array}{lll}.70633 & 38189 & 21\end{array}$ .873402727872 .975193834698

6 .224468995404 .409533350456 .597789048417 .768413604608 .901350733849 .980797208441

7

.183828768301 .340807595106 .507940524029 .670363410111 .812588465990 .920856417255 .984667450786 $b_{j}$

$\begin{array}{lll}.00007 & 7073707\end{array}$ .001447008789 .008926967613 .028547842753 .055224874164 .066021845935 .039754387040

.000029709230 .000598950038 .004077924135 .014909933450 .034719950661 .054910097291 .058000565268 .032752869927

.166666666667

$\begin{array}{lll}.03833 & 75627 & 37\end{array}$ .128329103930

.007297003638 .064596612296 .094773050733

$.00153 \quad 44797 \quad 48$ .021428404631 .072056364165 .071647418122

.000369715499 .006729690430 .033767744958 .070071339705 .055728176075

.000101325820 .002187925655 .013969653085 .041486346981 .064458859164 .044462555961

.000031104606 $.00075 \quad 5383832$ .005660413704 .020959298175 .045104981582 .057907613539 .036247871229 


\begin{tabular}{|c|c|c|c|}
\hline$m$ & $j$ & $x_{j}$ & $b_{j}$ \\
\hline 8 & 1 & .153150661610 & .000010531645 \\
\hline & 2 & .287264403884 & .0002783586 \\
\hline & 3 & .434627406699 & .0023353415 \\
\hline & 4 & .584518566563 & .01004461438 \\
\hline & 5 & $.7251264097 \quad 13$ & .02648530111 \\
\hline & 6 & .845189487931 & .045885653201 \\
\hline & 7 & $.9350435074 \quad 56$ & .0515342238 \\
\hline & 8 & .987460508524 & .0300926423 \\
\hline
\end{tabular}

The polynomials $q_{i}(x)$ are obtained by orthonormalization of the sequence $1, x, x^{2}, \cdots$ with weight function $x^{n}$ in the interval $(0,1)$. The Jacobi polynomials are defined by Courant and Hilbert [1] as follows:

$$
J_{m, n}=\frac{n !}{(m+n) !} x^{-n} \frac{d^{m}}{d x^{m}} x^{m+n}(1-x)^{m} .
$$

(Editor's note. The author's $J_{m, n}(x)$ is Courant's $G_{m}(n+1, n+1, x)$, and this, except for a normalizing factor, is $P_{m}^{(n, 0)}(2 x-1)$ of the Bateman Project [3].) Since [1] we have

$$
\int_{0}^{1} x^{n} J_{k, n} J_{m, n} d x=\frac{1}{(n+2 m+1)\left(\begin{array}{c}
m+n \\
n
\end{array}\right)^{2}} \delta_{k, m},
$$

the corresponding orthonormal sequence is

$$
\begin{aligned}
q_{m}(x) & =\sqrt{n+2 m+1}\left(\begin{array}{c}
m+n \\
n
\end{array}\right) J_{m, n}(x) \\
& =\sqrt{n+2 m+1} \sum_{k=0}^{m}(-1)^{k}\left(\begin{array}{c}
m+n+k \\
m
\end{array}\right)\left(\begin{array}{l}
m \\
k
\end{array}\right) x^{k}
\end{aligned}
$$

The explicit formula for $b_{j}$ is

$$
b_{j}=\left\{\sum_{i=0}^{m-1}\left[q_{i}\left(x_{j}\right)\right]^{2}\right\}^{-1}
$$

Within the range of values given in tables I, II, III of the Hammer, Marlowe, Stroud paper, it is seen that these polynomials are identical to those given therein except for a factor of -1 in the ones of odd degree associated with weight function $x^{2}$. Obviously, this has no effect on the values in the table.

The Newton-Raphson method with synthetic division was used to find the zeros. With the polynomials of higher degree (i.e., for $m=6,7$, and 8) and especially for the higher values of $n$ where the coefficients are large, the round-off errors in the zeros for 18 decimal places were seen to be considerable. As a check these preliminary values were substituted back into the original equation and the process was carried out again with the zeros recorded after each iteration. 
Synthetic division was not used this time because it was felt that the round-off error would be carried into the reduced polynomial. Observing the erratic property of the last digits it was concluded that the limit of accuracy in the worst case is about 12 decimal places. There seems to be no question for values of $m$ from 1 to 5 where $n=0,1$, and 2 .

A check of the $b_{j}$ was made through the fact that

$$
\sum_{j=1}^{m} b_{j}=\int_{0}^{1} x^{n} d x=\frac{1}{n+1}
$$

for each polynomial of degree $m$ associated with $x^{n}$. Here again the limit of accuracy is put at about the twelfth decimal place for the worst possible cases (i.e., with $m$ and $n$ both large). If one wishes to round off values to ten or eleven decimal places there should be no doubt of the accuracy.

I wish to acknowledge the helpful suggestions of the referee concerning the form of the first three paragraphs. The calculations were carried out in the Numerical Analysis Laboratory of the University of Wisconsin on the IBM Type 650. I am grateful for the use of the 18-digit floating decimal routine of Eugene Albright and his helpful suggestions in programming. The calculations and work were supported by funds of the Wisconsin Alumni Research Foundation granted by the Graduate Research Committee.

University of Wisconsin

Herbert Fishman

Madison, Wisconsin

1. R. Courant \& D. Hilbert, Methods of Mathematical Physics, First English edition, Translated and Revised from the German Original, Interscience Publishers, Inc., New York, v. 1, 1953, p. 90-91.

2. P. C. Hammer, O. J. Marlowe, \& A. H. Stroud, "Numerical integration over simplexes and cones," $M T A C$, v. 10, 1956, p. 130-137.

3. A. ERDELYI (editor), Higher Transcendental Functions, v. 2, McGraw-Hill Book Co., Inc., New York, 1953, p. 168-170.

4. William VernoN LovitT, Linear Integral Equations, McGraw'-Hill Book Co., Inc., New York, 1924.

\section{Tables of the Exponential Integral $\mathrm{Ei}(\mathbf{x})$}

In some molecular structure calculations it is desirable to have values of the integral $\mathrm{Ei}(x)$ to higher accuracy than is provided by the standard tables [1]. Direct computation of the values needed is extremely tedious over a wide range of the argument intermediate between the regions where expansions about zero and infinity are useful. However, if a net of sufficiently accurate values of $\mathrm{Ei}(x)$ has already been computed, then the generation of additional values intermediate between these becomes a problem of a considerably lesser magnitude. A few such values have been published $[2,3]$. Kotani $[4]$ has given a considerable number of values in his most valuable compilation, but unfortunately they are of somewhat fewer significant figures than under discussion here. A prohibitive amount of labor would be required to make such an extensive accurate table that only simple interpolation formulas need be used. However, a most useful intermediate objective could be achieved if a table were prepared with entries sufficiently closely 